\title{
Dermoscopic diagnosis of subungual hematoma: new observations
}

\author{
Mahmut Sami Metin¹, Ömer Faruk Elmas² \\ ${ }^{1}$ Department of Dermatology and Venereology, Batman Medical Park Hospital, Batman, Turkey \\ ${ }^{2}$ Department of Dermatology and Venereology, Faculty of Medicine, Ahi Evran University, Kırşehir, Turkey
}

Adv Dermatol Allergol 2020; XXXVII (4): 490-494

DOI: https://doi.org/10.5114/ada.2020.98235

\begin{abstract}
Introduction: There are very few studies focusing on the dermoscopic features of subungual hematoma which is one of the major imitators of subungual melanoma.

Aim: To identify the dermoscopic findings of subungual hematoma, which will facilitate the diagnostic process by reducing the use of more invasive diagnostic methods like nail avulsion or biopsy.

Material and methods: In this study, clinical and dermoscopic findings of the cases were reviewed. The diagnosis of subungual hematoma was confirmed by observing progression of the colour change to the distal edge of the nail plate in all the cases.

Results: A total of 47 subungual hematomas were enrolled in the study. The most common colour was purple-black (53\%). Blue-white colour was observed in $12(26 \%)$ lesions. $9(19 \%)$ lesions showed granular leukonychia. All of the lesions had a homogenous pattern. In 25 (53\%) lesions, a globular pattern was observed. 14 (30\%) lesions showed a streaks pattern. Peripheral fading and periungual haemorrhage were present in 14 (30\%) and 9 (9\%) lesions, respectively.

Conclusions: We detected two new findings which have not been described previously for subungual hematoma: the first one is "blue-white colour" which is known as an important clue to melanoma. The second one is granular leukonychia localized on the hematoma. We suggest that in any case of the nail discoloration, a thorough dermoscopic examination should be performed. Moreover, progression of the colour change to the distal edge should be observed to ensure that a possible melanoma is not overlooked.
\end{abstract}

Key words: dermoscopy, hematoma, subungual.

\section{Introduction}

Subungual hematoma ( $\mathrm{SH}$ ) can be described as blood accumulation between the nail bed and nail plate. It is usually caused by a sudden painful trauma or repetitive micro traumas [1, 2]. SH usually presents with a sharpedged colour change varying from red to black under the nail plate. The diagnosis is usually not difficult if the progression of the hematoma to the distal edge can be observed [3]. However, extraordinary clinical appearance and colours in some cases may cause difficulty in diagnosis [4]. In such conditions, it may be necessary to perform some interventions like nail avulsion or biopsy to confirm the diagnosis. Subungual melanoma is the main differential diagnosis of SH which has to be excluded. Dermoscopy is an effective and practical diagnostic tool in everyday dermatological practice. It has been shown that dermoscopy provides important clues to different causes of colour changes in nails [5, 6]. However, there are few studies focusing on the dermoscopic features of $\mathrm{SH}[1,7]$.

\section{Aim}

Here, we aimed to identify the dermoscopic findings of $\mathrm{SH}$ which will facilitate the diagnostic process by reducing the use of more invasive methods in the diagnostic process.

\section{Material and methods}

Patients with an approved diagnosis of subungual hematoma at a tertiary university hospital were enrolled in the study. Clinical, demographic and dermoscopic findings of the cases were retrospectively reviewed. Only high quality dermoscopic images were included in the study for the retrospective analysis. The diagnosis of sub-

Address for correspondence: Ömer Faruk Elmas, Department of Dermatology and Venereology, Faculty of Medicine, Ahi Evran University, Kırşehir 40000, Turkey, phone: +90 5330260679, e-mail: omerfarukmd@gmail.com Received: 30.10.2018, accepted: 12.12.2018. 
ungual hematoma was confirmed by observing progression of the colour change to the distal edge of the nail plate in all cases. Dermoscopic imaging was performed with a handheld dermoscope with $10 \times$ magnification (Dermlite II ProHR, 3GEN, San Juan Capistrano, California, USA) and a dermoscope adopted high resolution mobile camera phone (iPhone 7 plus, Apple, California, USA). $2 \times$ optical zoom was also used for more magnification. All the procedures followed the Helsinki Declaration and the study was approved by the local clinical research ethics committee.

\section{Statistical analysis}

Descriptive statistical analysis was performed using SPSS package program (SPSS Inc., Chicago, IL).

\section{Results}

\section{Clinical and demographic findings}

Forty-one patients with a total of 47 lesions of subungual hematoma were enrolled in the study. The mean age of the patients was 45 (age range: 9-67) and 29 (72.5\%) of the patients were men. The mean duration of the lesions was 8 weeks (duration range: 3 days -5 months). The most common localization was the big toe. Two nails in 5 patients and 3 nails in 2 patients were affected. A single nail was affected in the remaining cases. The most frequent site of the hematoma was proximal third of the nail ( $n=23,49 \%)$. The other sites were central
( $n=10,21 \%)$ and distal two third $(n=7,15 \%)$ of the nail. Diffuse involvement was observed in 7 (15\%) nails. Thirty (64\%) patients gave a history of trauma. The lesion was painful on palpation in $8(18 \%)$ cases. A microbiologically confirmed diagnosis of distal subungual onychomycosis was made for 8 nails with subungual hematoma. No more invasive intervention was needed to confirm the diagnosis of $\mathrm{SH}$ in any patients.

\section{Dermoscopic findings}

\section{Colour}

Forty-two (89\%) nails showed more than one colour. The most frequent colour we observed was purple black $(n=25,53 \%)$ (Figures 1 A, B, 2 A). Numbers and frequencies of the other colours were as follows: red black ( $n=15$, $32 \%)$ (Figure $2 \mathrm{~A})$, brown $(n=13,28 \%)$ (Figure $2 \mathrm{~B})$, black $(n=13,28 \%)$ (Figure $2 \mathrm{~B})$, blue white $(n=12,26 \%)$ (Figures $2 \mathrm{~B}, 3 \mathrm{~A})$, red $(n=10,21.2 \%)$ (Figures $1 \mathrm{~B}, 3 \mathrm{~B})$, grey black $(n=7,17 \%)$ (Figure $2 \mathrm{~B})$ and purple $(n=3,6 \%)$ (Figures 1 A, B). Granular leukonychia on the hematoma was observed in 9 (19\%) nails (Figure 3 B).

Distribution of the colours detected is summarized in Table 1.

\section{Pattern}

The homogeneous pattern was observed in all the nails (100\%) (Figures 1 A, B, 2 A). The globular pattern (Figures 1 B, $2 \mathrm{~A}, 3 \mathrm{~B}$ ) and streaks pattern (Figures $1 \mathrm{~A}, 3 \mathrm{~A}$ ) were observed in 25 (53\%) and 14 (30\%) nails, respectively. Twen-
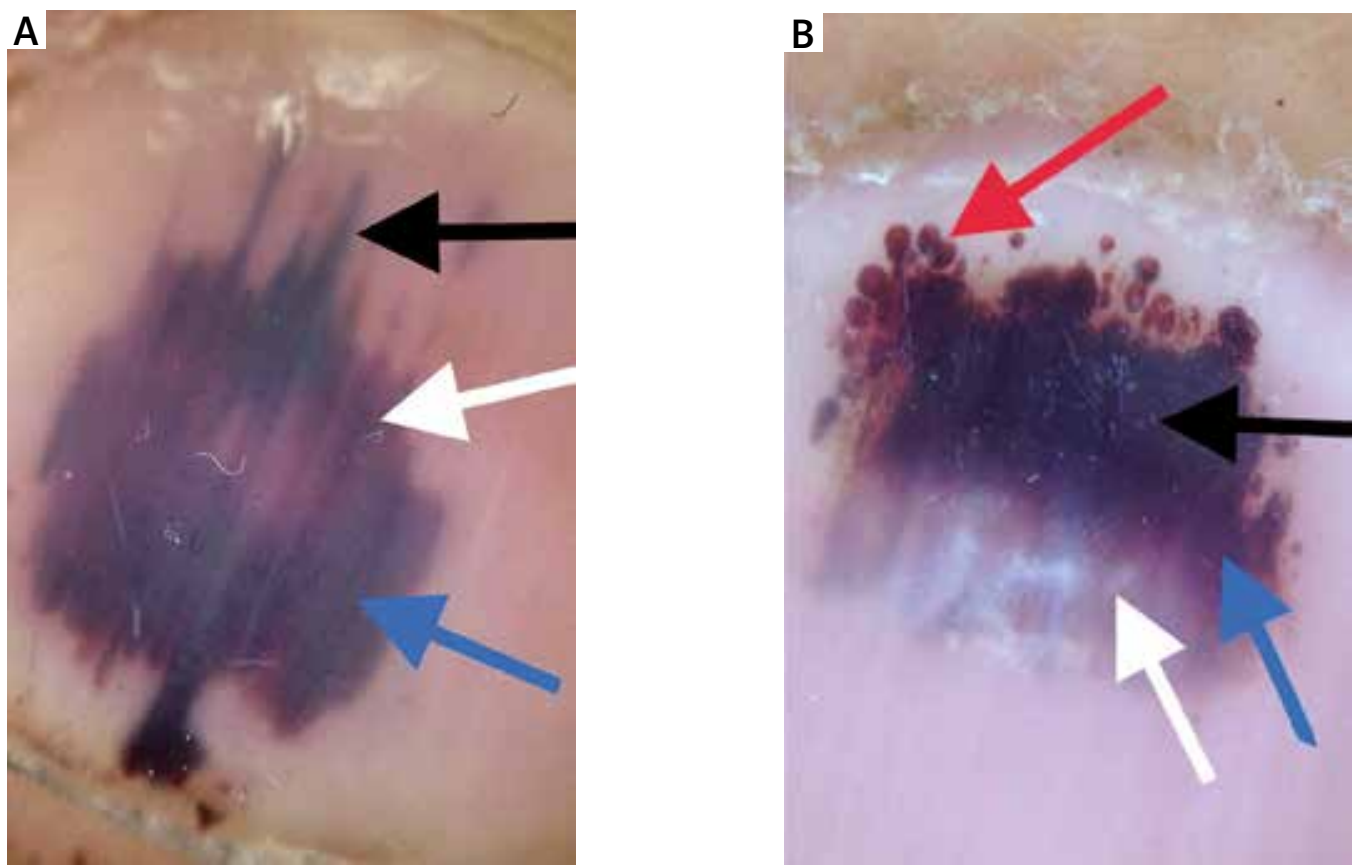

Figure 1. A - Homogeneous pattern with purple black (blue arrow) and purple (white arrow) colours, streaks pattern with black colour (black arrow), B - homogenous pattern with purple black (black arrow) and purple (blue arrow) colours. Globular pattern with red colour (red arrow). Peripheral fading (white arrow) 

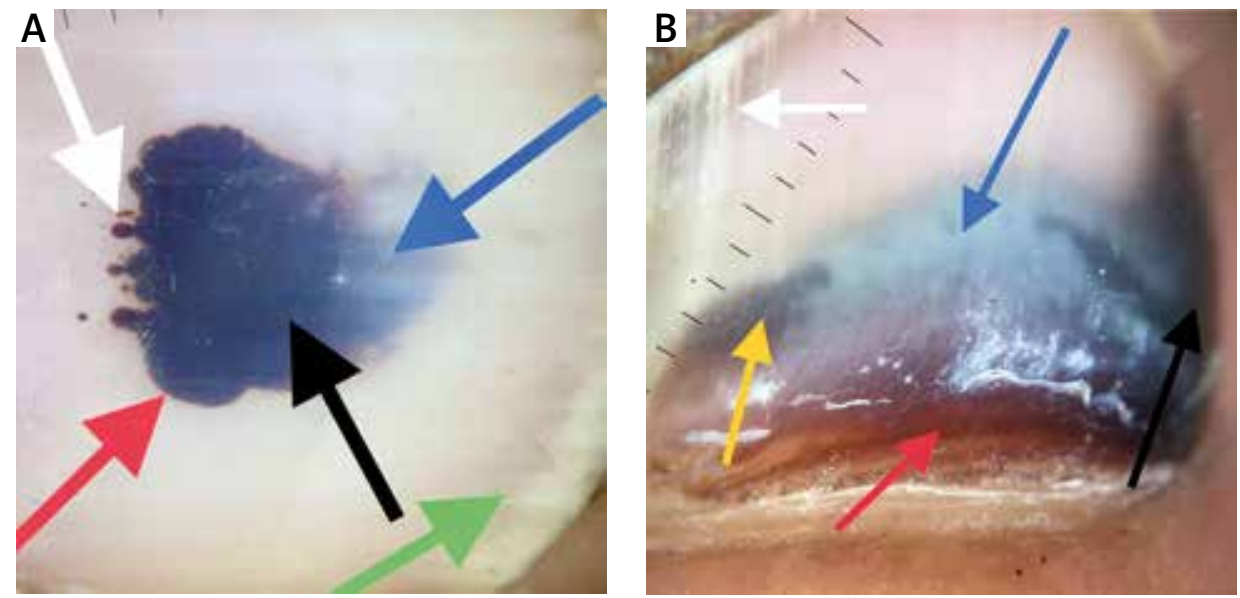

Figure 2. A - Homogenous pattern with purple black colour (black arrow), sharp edge (red arrow), globular pattern with red black colour (white arrow), peripheral fading (blue arrow), white jagged edges (green arrow), B - homogenous pattern with brown (red arrow), grey (yellow arrow), black (black arrow) and blue white (blue arrow) colours, white longitudinal striae (white arrow)
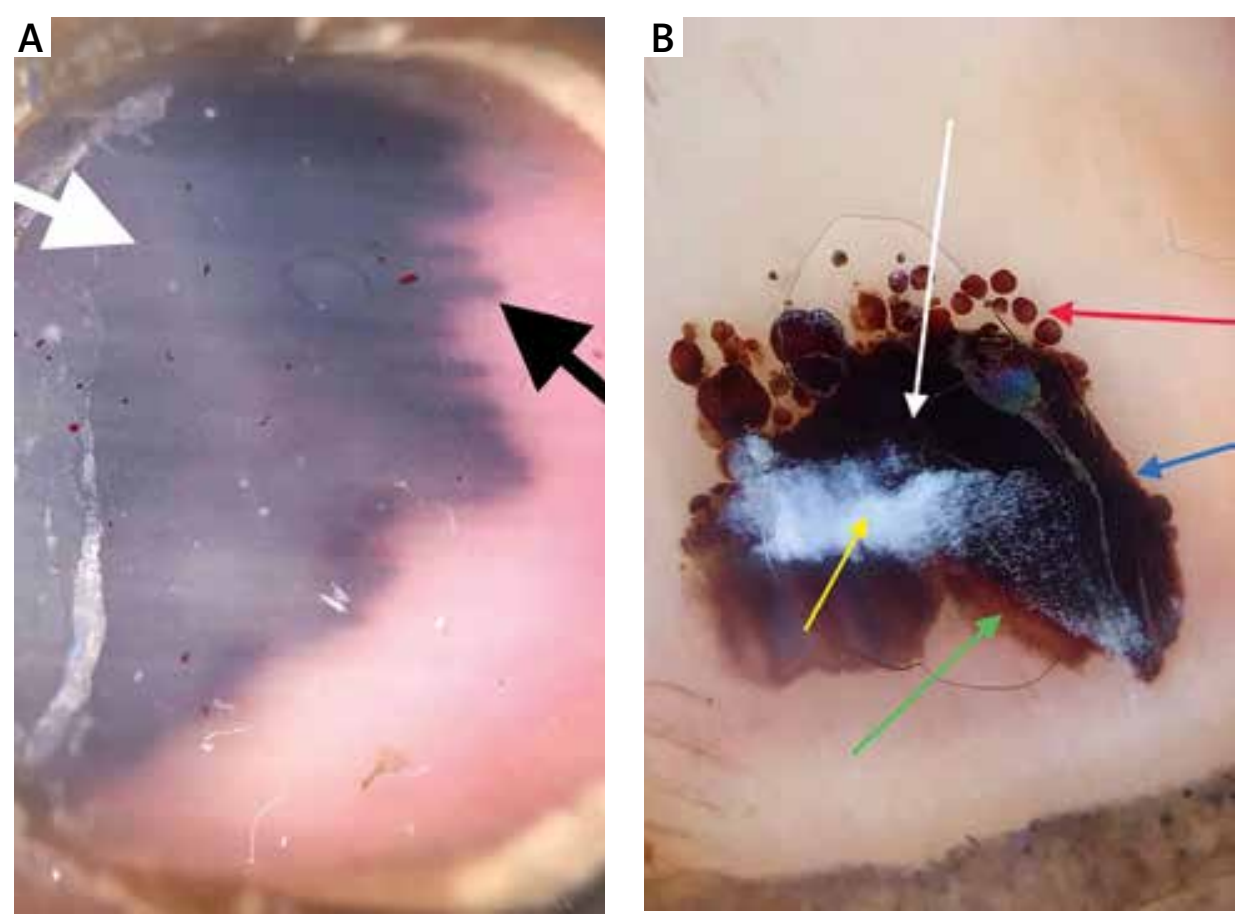

Figure 3. A - Homogenous pattern with blue white (white arrow) and streaks pattern with purple black (black arrow) colours, B - homogeneous pattern with black colour (white arrow), globular pattern with red colour (red arrow), sharp edge (blue arrow), peripheral fading with red colour (green arrow). Granular leukonychia (yellow arrow)

ty-seven (57\%) nails showed at least a combination of two patterns. The homogenous pattern was the only pattern in 20 (43\%) nails.

A combination of three patterns was observed in 10 (21\%) nails. Twelve (26\%) nails showed a combination of homogenous and globular patterns. Five (11\%) nails showed a combination of homogenous and streaks patterns. Peripheral fading (Figures 1 B, 2 A, 3 B) and periungual haemorrhage were observed in 14 (30\%) and 9 (19\%) nails, respectively.
Dermoscopic patterns of the cases are summarized in Table 2.

\section{Accompanying dermoscopic findings}

White longitudinal striae were observed in 6 (13\%) nails (Figure 2 B). Five (11\%) lesions showed onycholysis. We also observed onychodystrophy and white jagged edges (Figure $2 \mathrm{~A}$ ) in $5(11 \%)$ and 4 (9\%) nails, respectively. 
The accompanying dermoscopic findings are summarized in Table 3.

\section{Discussion}

$\mathrm{SH}$ is a frequently encountered entity in the everyday dermatological practice and is one of the mimickers of subungual melanoma [1]. When the literature is reviewed, it seems that there are many studies investigating the role of dermoscopy in diagnosis of different nail diseases. However, few original studies focused on the dermoscopic findings of $\mathrm{SH}[1,8]$.

Dermoscopic findings of the entity were initially described in the study of Ronger et al. [9]. In another study, Öztaş followed the clinical and dermoscopic progression of the early subungual hematomas [8]. Until now, the most comprehensive study focused on the subject has been performed by Mun et al., in which dermoscopic findings of subungual hematomas were described in detail. Mun et al. stated that $88 \%$ of the cases were not diagnosed accurately in the primary or secondary hospital and were referred to their dermatology clinic at a third-level hospital for further evaluation [1]. In the present study, 17 (41\%) patients were referred to our tertiary centre from primary and secondary hospitals.

In Mun et al.'s study, the most common dermoscopic pattern was the homogenous pattern followed by globular and streaks patterns similar to our study [1]. A combination of at least two patterns was observed in $53 \%$ of the cases [1]. This rate was $57 \%$ in our study.

The pathogenesis of homogeneous and globular patterns can be explained by the direct effect of a trauma, however, the pathogenesis of the streaks pattern has been a subject of curiosity. Sato et al. suggested that the streaks pattern is associated with the specific longitudinal microvascular anatomy of the nail bed regarding their experimental case study [10].

Mun et al. described two new findings in their study: periungual haemorrhage and peripheral fading [1]. In our study, $36 \%$ and $38 \%$ of the nails showed periungual haemorrhage and peripheral fading, respectively.

In the present study, the most common colour was purple black (53\%) followed by red black (32\%). Mun et al. observed purple black and red black colours in $37 \%$ and $16 \%$ of the nails, respectively [1].

In the present study we observed blue white colour in $12(26 \%)$ nails. To the best of our knowledge, this colour has not been described previously for SH. Blue white colour change was localized on the proximal third of the nail in 9 nails and in distal two thirds in 3 nails.

Another novel finding which we identified was granular leukonychia. Leukonychia is usually caused by nail plate abnormalities and as opposite to hematoma it is not localized on the nail bed [11]. There were 3 main features of leukonychia we observed: 1) It was localized on
Table 1. Distribution of the colours in subungual hematoma

\begin{tabular}{lc}
\hline Colours & Number (\%) \\
\hline Purple black & $25(53)$ \\
\hline Red black & $15(32)$ \\
\hline Brown & $13(31)$ \\
\hline Black & $13(31)$ \\
\hline Blue white & $12(26)$ \\
\hline Red & $10(21)$ \\
\hline Grey black & $7(15)$ \\
\hline Purple & $3(6)$ \\
\hline Granular leukonychia & $9(19)$ \\
\hline
\end{tabular}

Table 2. Distribution of the dermoscopic pattern in subungual hematoma

\begin{tabular}{lc}
\hline Pattern & Number (\%) \\
\hline Homogenous & $47(100)$ \\
\hline Globular & $26(55)$ \\
\hline Streaks & $17(36)$ \\
\hline
\end{tabular}

Table 3. Distribution of the accompanying dermoscopic findings

\begin{tabular}{lc}
\hline Dermoscopic finding & Number (\%) \\
\hline Granular leukonychia & $9(19)$ \\
\hline Periungual haemorrhage & $9(19)$ \\
\hline White longitudinal striae & $8(17)$ \\
\hline Thickening of nail plate & $7(41)$ \\
\hline Onychodystrophy & $5(11)$ \\
\hline Onycholysis & $5(11)$ \\
\hline White jagged edges & $4(9)$ \\
\hline
\end{tabular}

a relatively large area and it was clinically visible. 2) It appeared weeks after hematoma. 3) It showed granular distribution on dermoscopic examination.

The exact etiopathogenesis of leukonychia is unknown, however, it is thought that immature parakeratotic corneocytes in the nail plate reflect light due to keratohyaline granules and thus, a white appearance occurs [11]. We also suggest that hematoma inhibits the maturation of corneocytes by the pressure on the ventral face of the nail plate and causes leukonychia.

Unlike the other studies on the subject, we also evaluated accompanying dermoscopic findings associated with onychomycosis. White longitudinal striae and white jagged edges were observed in $6(13 \%)$ and $4(9 \%)$ nails, respectively. Mycological examination allowed a diagnosis of onychomycosis in 8 nails. Five out of these 8 nails showed longitudinal striae and the remaining 3 showed white jagged edges. Piraccini et al. 
showed that the presence of white longitudinal striae and white jagged edges is a pretty sensitive and specific dermoscopic finding for onychomycosis [12].

In the present study, we also observed onycholysis in $5(10.6 \%)$ nails and dystrophic appearance in 5 (11\%) nails. $\mathrm{KOH}$ preparation results were negative for these nails. Other possible causes of onycholysis including cutaneous diseases, systemic conditions and drugs were also not observed in any nail with onycholysis. Therefore, trauma was considered to be the most probable cause of these findings.

Lack of a control group and the retrospective nature of the study are the main limitations which have to be pointed out. However, there are some points that need to be emphasized regarding the results of the study:

There are very few studies focusing on the dermoscopic findings of $\mathrm{SH}$, which is considered to be one of the imitators of subungual melanoma. The dermoscopic findings of $\mathrm{SH}$ which we observed supported the results of previous studies.

Herein, we identified a novel finding which we called granular leukonychia. We hypothesized that this findings is associated with the blockage of corneocyte maturation on the ventral surface of the nail plate due to hematoma caused pressure.

Another novel finding we identified was blue white colour change which we observed in $12 \%$ of the lesions. Blue white colour is known as the main dermoscopic finding of cutaneous melanoma [13]. In case of the presence of this colour change, we suggest investigating the presence of other dermoscopic characteristics of subungual hematoma and monitoring the patient closely.

In the present study, we observed the dermoscopic findings leading to the diagnosis of onychomycosis. Mycological examination allowed a diagnosis of onychomycosis in $8(18 \%)$ nails. Considering the prevalence of onychomycosis in the population, it can be suggested that nails with onychomycosis have a greater tendency to trauma resulting in hematoma than normal nails [14-16]. It is obvious that in order to confirm this proposal, more studies involving a larger sample size are needed.

It should be kept in mind that subungual hematoma may be associated with underlying neoplastic conditions. After a careful dermoscopic examination, all cases should be monitored to prove that hematoma progresses distally. In our study, all the affected nails were followed 6 weeks on average and the diagnosis of $\mathrm{SH}$ was confirmed.

\section{Conflict of interest}

The authors declare no conflict of interest.

\section{References}

1. Mun JH, Kim GW, Jwa SW, et al. Dermoscopy of subungual haemorrhage: its usefulness in differential diagnosis from nail-unit melanoma. Br J Dermatol 2013; 68: 1224-9.

2. Pierre M. The Nail. $1^{\text {st }}$ edn. Churchill Livingstone, Edinburgh, New York 1981.

3. Braun RP, Baran R, Le Gal FA, et al. Diagnosis and management of nail pigmentations. J Am Acad Dermatol 2007; 56: 835-47.

4. Zaias N. The Nail in Health and Disease. $2^{\text {nd }}$ edn. Appleton \& Lange, Norwalk, Connecticut 1990.

5. Benati E, Ribero S, Longo C, et al. Clinical and dermoscopic clues to differentiate pigmented nail bands: an International Dermoscopy Society study. J Eur Acad Dermatol Venereol 2017; 31: 732-6.

6. Piraccini BM, Dika E, Fanti PA. Tips for diagnosis and treatment of nail pigmentation with practical algorithm. Dermatol Clin 2015; 33: 185-95.

7. Chieb S, Baha H, Hali F. Subungual hematoma: clinical appearance of resolution over time. Dermatol Online J 2015; 21: 13030/qt689871cb.

8. Oztas MO. Clinical and dermoscopic progression of subungual hematomas. Int Surg 2010; 95: 239-41.

9. Ronger S, Touzet S, Ligeron C, et al. Dermoscopic examination of nail pigmentation. Arch Dermatol 2002; 138: 1327-33.

10. Sato T, Tanaka M. The reason for red streaks on dermoscopy in the distal part of a subungual hemorrhage. Dermatol Pract Conceptual 2014; 4: 83-5.

11. Tosti A, Piraccini BM. Nail disorders. In: Dermatology. Bolognia JL, Jorizzo JL, Rapini RP (eds.). Mosby, Philadelphia 2003; 1061-78.

12. Piraccini BM, Balestri R, Starace M, Rech G. Nail digital dermoscopy (onychoscopy) in the diagnosis of onychomycosis. J Eur Acad Dermatol Venereol 2013; 27: 509-13.

13. Armengot-Carbó M, Nagore E, García-Casado Z, BotellaEstrada R. The association between dermoscopic features and BRAF mutational status in cutaneous melanoma: significance of the blue-white veil. J Am Acad Dermatol 2018; 78: 920-6.e4.

14. Sahin I, Oksuz S, Kaya D, et al. Dermatophytes in rural area of Duzce Turkey. Mycoses 2004; 47: 470-4.

15. Sigurgeirsson B, Baran R. The prevalence of onychomycosis in the global population: a literature study. J Eur Acad Dermatol Venereol 2014; 28: 1480-91.

16. Bristow IR, de Berker DA, Acland KM, et al. Clinical guidelines for the recognition of melanoma of the foot and nail unit. J Foot Ankle Res 2010; 3: 25. 